\title{
A three-dimensional model for the probabilistic intergranular failure of polycrystalline arrays
}

\author{
D G Harlow†, H-M Luł, J A Hittinger§, T J Delph† and R P Wei† \\ $\dagger$ Department of Mechanical Engineering and Mechanics, Lehigh University, 19 Memorial Drive \\ West, Bethlehem, PA 18015-3085, USA \\ $\ddagger$ Department of Mechanical and Aerospace Engineering, West Virginia University, Morgantown, \\ WV 26506-6101, USA \\ $\S$ Department of Mechanical and Aerospace Engineering, University of Michigan, Ann Arbor, \\ MI, USA
}

Received 6 November 1995, accepted for publication 18 February 1996

\begin{abstract}
A three-dimensional grain model, in which the grains are represented by regular truncated octahedra, has been developed to study probabilistic time-dependent intergranular failure in polycrystalline arrays. In this model, grain boundary facets are assumed to fail randomly in time, as a function of the facet normal stress. A simple approximate method for calculating the load shed by failed facets and a reasonable choice of failure criterion complete the model. This leads to a conceptually simple, but computationally complex, model capable of handling assemblages consisting of relatively large numbers $(>5000)$ of grains. The predicted scatter in the times-to-failure and the variation in number of failed facets with time are in quite reasonable agreement with available experimental data.
\end{abstract}

\section{Introduction}

Experimental evidence has consistently indicated that time-dependent intergranular failure processes, typically at elevated temperatures, are characterized by some degree of randomness. This is reflected not only in the scatter in the time-to-failure data for uniaxial stress creep tests [1-4], but also in the randomness present in measures of internal damage such as intergranular creep cavitation [5]. Accordingly, there have been a number of recent efforts to construct microscopic models of intergranular failure which contain one or more random features, e.g. [6-11]. As has been the case with the vast majority of microscopic models, most of these efforts have been confined to a consideration of simple bicrystal structures. There have, however, been recent efforts to consider more realistic grain structures; e.g. the work of Riesch-Oppermann and Brückner-Foit [10], who considered the growth of creep cavitation in an irregular, two-dimensional rigid grain structure, and that of van der Giessen and Tvergaard [11], who investigated a similar problem in an irregular, creeping assemblage of grains, with random cavity nucleation.

It is known, however, that intergranular failure processes in three dimensions possess a higher degree of complexity than can be adequately represented by models involving simple one-, or even two-dimensional grain structures. Anderson and Rice [12], who investigated the growth of constrained intergranular cavitation in a regular three-dimensional grain structure, found significant quantitative differences between the results of their work 
and previous work involving two-dimensional grain structures $[13,14]$. Accordingly, the purpose of this paper is to consider a model of three-dimensional intergranular failure containing one or more random features.

\section{Description of the model}

\subsection{Grain model}

One of the most significant difficulties in simulating three-dimensional intergranular failure is the modelling of the grain structure. A common method is to model grains as the random division of space into subsets, i.e. to represent the grain structure by a random tessellation of two- or three-dimensional space. This approach can yield results that, at least in two dimensions, have a striking resemblance to actual grain structures observed on polished surfaces. In two dimensions, such structures can be computationally generated by one of many algorithms, e.g. the Voronoi (Dirichlet) tessellation [15-20] or the JohnsonMehl model $[15,18]$. There exist three-dimensional versions of these random, twodimensional tessellations $[15,18]$, but the associated algorithms are often quite difficult to implement [19].

In a well annealed metal, the grains are all roughly of the same shape and size. This suggests that a homogeneous crystalline material can be reasonably well represented by a three-dimensional, regular tessellation of identical convex polyhedra. There are only two regular or semi-regular polyhedra that can completely fill space in a regular tessellation: the cube and the fourteen-sided truncated octahedron, sometimes also known as the WignerSeitz cell. Of these two choices, the truncated octahedron, shown in figure 1, better represents actual multifaceted grains. Consequently, it has been used in previous studies involving three-dimensional grain structures, e.g. [12,21]. For the present study, this polyhedron was also chosen to represent the grains.

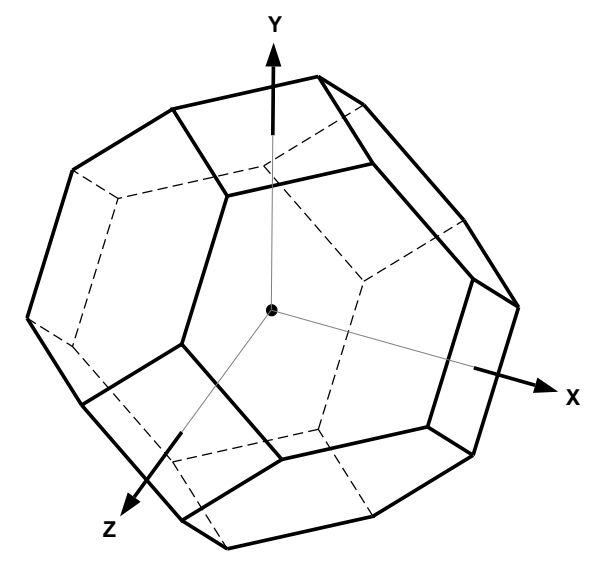

Figure 1. Fourteen-sided truncated octahedron.

Numerically, the truncated octahedron may be specified by eighteen independent values, assuming a constant effective diameter. Three values are needed to represent the coordinate triple of the centroid. The remaining fifteen values are pointers, which designate the grain itself and its fourteen neighbours. Using this system of representation, it is easy to generate numerically grain assemblages of any size, limited only by available computer memory. In 


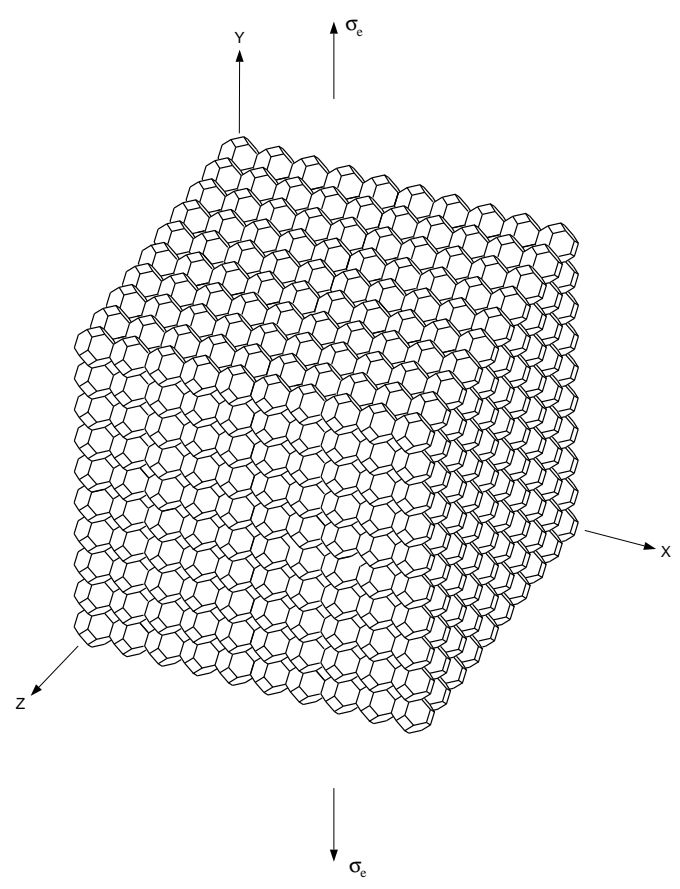

Figure 2. Cubic matrix of $1729\left(10^{3}+9^{3}\right)$ grains, and its orientation with respect to a set of Cartesian coordinate axes.

the present work, a perfectly packed cubic matrix of 1729 grains $\left(10^{3}+9^{3}\right)$ was utilized for most studies, along with a limited series of simulations on matrices of smaller and larger sizes. Figure 2 shows a typical assemblage of 1729 grains.

\subsection{Grain boundary failure model}

At high temperatures, a dominant mechanism for intergranular failure in polycrystalline materials is cavitation. The growth and coalescence of these cavities lead to the formation of microcracks, which then grow to such a size as to result in eventual failure. Because detailed simulations of the growth of large numbers of intergranular cavities is beyond present computational capabilities, at least in three dimensions, a simplified model for grain boundary failure will be considered instead. The basic assumption of the model is that grain boundary facets fail instantaneously at random times, presumably in response to the evolution of grain boundary damage, to form facet-sized cavities, or microcracks. This assumption has some support from models of constrained creep cavitation [22], which indicate that cavitated grain boundary facets tend to shed load, and to behave as facet-sized microcracks, at creep strain rates typical of engineering applications.

Most models for the growth of creep cavitation assume that the grain boundary normal stress is the single stress component which influences the cavity growth and that the effects of shearing stresses are negligible. Nix et al [23] have discussed the importance of the facet normal stress in the creep rupture process. A similar assumption will be made here. Furthermore, any effects of grain boundary sliding or grain deformability upon the facet normal stresses will be ignored. The simple loading shown in figure 2, which consists of a constant uniaxial stress of magnitude $\sigma_{e}$ applied in the $y$-direction normal to the square 
faces of the truncated octahedra will be considered. For this loading condition only the horizontal and oblique facets will experience normal stresses.

One of the key elements in the model is the cumulative distribution function (CDF) for the time-to-failure of a grain boundary facet. Ideally, this should be estimated from a mechanistically based model for the evolution of cavity growth, in conjunction with experimental data. This, however, represents a difficult undertaking, and the experimental data which would support such a model are, by-and-large, lacking. Failing this, a form for the CDF will be assumed which has some degree of experimental support. The CDF for the time-to-failure of a facet is taken to be

$$
F(t)=1-\exp \left[-\Psi\left(\int_{0}^{t} \kappa(\sigma(s)) \mathrm{d} s\right)\right] \quad t \geqslant 0
$$

where $\sigma(\bullet)$ is interpreted as the normal stress history acting on the facet over the entire life of the facet, $\kappa(\bullet)$ is the breakdown rule which describes the accumulation of damage, and $\Psi(\bullet)$ is the hazard function which describes the reliability behaviour. Both $\kappa(\bullet)$ and $\Psi(\bullet)$ must be increasing, unbounded, and continuous functions. Furthermore, $\Psi(0)=0$. The stress $\sigma(\bullet)$ is arbitrary, and, in the present study, will be piecewise constant with time.

The CDF given by (1) is quite general and has a wide range of applications. In fact, it can be considered to be the underlying CDF for an inhomogeneous Poisson process [24]. Coleman [25] was one of the first to use it in engineering applications, and many authors have subsequently made use of it in various forms. Phoenix [26] has given a good survey of the development and applicability of (1). Frequently, $\Psi(\bullet)$ is assumed to be the Weibull hazard function given by

$$
\Psi(x)=x^{\alpha} \quad x \geqslant 0 \quad \alpha>0
$$

with values of $\alpha$ typically lying between 0.25 and 5.00. The most popular breakdown rule is the power-law form:

$$
\kappa(x)=\left(\frac{x}{\gamma}\right)^{\rho} \quad x \geqslant 0 \quad \gamma>0 \quad \rho>0
$$

where $\rho$ ranges from 1 to 80 in applications. In analysing the results of creep rupture experiments with copper bicrystals, Farris et al [3] showed that the CDF for the time-tofailure for a single grain boundary could be well approximated by (1) with the Weibull hazard function (2) and the power-law breakdown rule (3). It thus seems reasonable to adopt this form for the present study. Hence, specifically, the CDF (1) for grain boundary facet time-to-failure will be

$$
F(t)=1-\exp \left\{-\left[t\left(\frac{\sigma}{\gamma}\right)^{\rho}\right]^{\alpha}\right\} .
$$

Farris et al [3] measured values of $\rho$ slightly in excess of one for grain boundary failure in copper bicrystals. It appears, however, that considerably higher values hold in polycrystalline materials which obey the Monkman-Grant relation. The parameter $\alpha$ is a measure of the statistical scatter in the grain boundary failure times.

In order to simplify the ensuing calculations in the model for polycrystalline materials, a transformation used previously by Phoenix and Tierney [27] is introduced. Let $Z_{w}, w=\mathrm{h}$ (horizontal) or o (oblique), be a random variable (RV) associated with each facet, which has the standard exponential $\mathrm{CDF}$, i.e.

$$
F_{Z_{w}}(z)=1-\exp \{-z\} \quad z \geqslant 0 .
$$


Let $T_{w}, w=\mathrm{h}$ or $\mathrm{o}$, be the $\mathrm{RV}$ for the transformed time-to-failure of a facet, defined in terms of the RV $Z_{w}$ by

$$
\Psi\left(\int_{0}^{T_{w}} \kappa\left(\sigma_{w}(s)\right) \mathrm{d} s\right)=Z_{w} .
$$

Note that $\Psi$ is an increasing function of $T_{w}$. Hence, using standard transformations

$$
\begin{aligned}
F_{T_{w}}(t) & =\operatorname{Pr}\left\{T_{w} \leqslant t\right\}=\operatorname{Pr}\left\{\Psi\left(\int_{0}^{T_{w}} \kappa\left(\sigma_{w}(s)\right) \mathrm{d} s\right) \leqslant \Psi\left(\int_{0}^{t} \kappa\left(\sigma_{w}(s)\right) \mathrm{d} s\right)\right\} \\
& =\operatorname{Pr}\left\{Z_{w} \leqslant \Psi\left(\int_{0}^{t} \kappa\left(\sigma_{w}(s)\right) \mathrm{d} s\right)\right\}=F_{Z_{w}}\left(\Psi\left(\int_{0}^{t} \kappa\left(\sigma_{w}(s)\right) \mathrm{d} s\right)\right) \\
& =1-\exp \left[-\Psi\left(\int_{0}^{t} \kappa\left(\sigma_{w}(s)\right) \mathrm{d} s\right)\right]=F_{T_{f}}(t)
\end{aligned}
$$

by (1). This implies that the use of independent and identically distributed (IID) standard exponential RVs $Z_{w}$ yields a probability model equivalent to (1) for facet time-to-failure. The advantage of this transformation is that, while the transformed times-to-failure $T_{w}$ are highly statistically dependent due to the local interactions between failed and unfailed facets, the standard representative RVs $Z_{w}$ are completely independent.

As will be described in detail in the next section, the failure of adjacent facets results in increases in load upon a given facet. Let $i_{1}, i_{2}, \ldots, i_{j}$ designate failed facets which cause an increase in load on facet $i$. As a consequence of the fact that the facet stresses are piecewise constant in time, the adjusted failure time for a facet resulting from successive increases in loads from adjacent failed facets may be obtained from (6), and is given by

$T_{w}\left(i \mid i_{1}, i_{2}, \ldots, i_{j}\right)=T_{i_{j}}+\left[\Psi^{-1}\left(Z_{i}\right)-\sum_{a=1}^{j}\left(T_{i_{a}}-T_{i_{a-1}}\right) \kappa\left(\sigma_{i_{a}}\right)\right]\left[\kappa\left(\sigma_{i_{j+1}}\right)\right]^{-1}$

where $T_{i_{a}}$ is the time-to-failure for facet $i_{a}$ which directly affects the time-to-failure of facet $i$, $T_{i_{0}}$ is identically zero, and $\sigma_{i_{a}}$ is the normal stress acting on facet $i$ during the period between the failure of facet $i_{a-1}$ and that of facet $i_{a}$. Note that the term $\sum_{a=1}^{j}\left(T_{i_{a}}-T_{i_{a-1}}\right) \kappa\left(\sigma_{i_{a}}\right)$ can be considered to represent the state of damage on facet $i$ at time $T_{i_{j}}$.

\subsection{Load-sharing rule}

In order to implement the probability model, the normal stress history for each grain boundary facet is required. In general, the normal stress on a facet will increase as a consequence of the failure of neighbouring facets. Prior to any facet failures and in the absence of grain boundary sliding, the normal stress across each facet will be just the component of the applied uniaxial stress field normal to the grain boundary facet, i.e. $\sigma_{w}=\sigma_{\mathrm{e}} \cos ^{2} \phi_{w}, w=\mathrm{h}$ or o, where $\phi_{w}$ is the angle between the facet normal and the $y$-axis. For the particular geometry and loading condition of this problem, $\phi_{\mathrm{h}}=0$ and $\phi_{\mathrm{o}}=\arccos (\sqrt{3} / 3)$ which implies that $\sigma_{\mathrm{h}}=\sigma_{\mathrm{e}}$ and $\sigma_{\mathrm{o}}=\sigma_{\mathrm{e}} / 3$. However, as facets begin to fail randomly, the stress distribution inside the assemblage becomes so complicated as to make detailed calculations extremely difficult. Hence, a simplified stress redistribution rule is proposed, which is quite similar in spirit to the load-sharing rules used in random failure models for fibrous composite materials, e.g. [28-30].

It is clear that the load shed from a failed grain boundary facet will be redistributed over some distance from the failed facet. However, it seems reasonable to believe that the bulk of the redistributed load will be carried by the facets bordering the failure area. In the 
interest of simplicity, the entire effects of load redistribution are assumed to be confined to the unfailed facets immediately adjacent to the failure area. The phrase failure area is used here to describe both an isolated failed facet and a contiguous region of failed facets. Based on this, the following load redistribution rules will be assumed:

(1) The load parallel to the loading axis carried by each failed grain boundary facet will be distributed equally, with certain exceptions, to its adjacent facet area. Since a larger facet can carry a larger load, the load redistributed to each adjacent facet will be

$$
\Delta P_{i_{j}}=P_{i}\left(\frac{A_{i_{j}}}{\sum_{j} A_{i_{j}}}\right)
$$

where $P_{i}$ is the load originally carried by the failed facet $i, i_{j}$ designates the $j$ unfailed facets adjacent to facet $i$ which share the load $P_{i}$, and $A_{i_{j}}$ is the projected area of the adjacent facet $i_{j}$ on the plane normal to the loading axis. If any of the adjacent facets have already failed, the load that would have been shared by that facet will be further redistributed to its adjacent facets.

(2) Exceptions to the load redistribution rule described above arise from the experimental observation that the preferred direction of intergranular crack propagation is normal to the loading axis. Hence, cracks do not tend to propagate in the direction of the loading axis and, more generally, do not tend to double back on themselves, i.e. do not propagate through an angle of greater than $\pm 90^{\circ}$ from a plane normal to the loading axis. In order to incorporate these observations into the load-sharing rule described above, loads are not redistributed to facets parallel to the loading axis, or to facets which, if they were to fail, would result in a reversal of the direction of crack propagation.

As a consequence of the fact that the remote stress is constant in time, the facet stresses are piecewise-constant functions of time. Jumps in facet stress occur when adjacent facets fail, with the magnitude of the jump being given by the load redistribution rules above. Implicit in these rules is the assumption that the facet stresses are not affected by deformation processes or by grain boundary sliding.

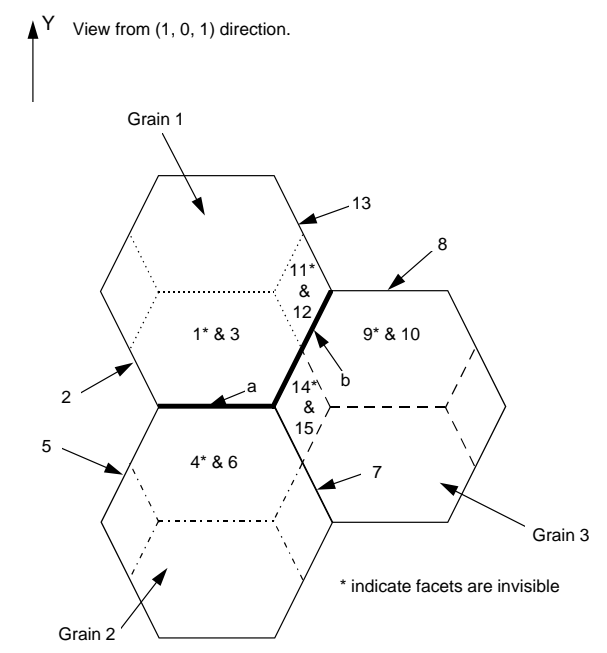

Figure 3. Simple example consisting of three grains to illustrate load-sharing rules.

These load redistribution rules are quite simple, but their computational implementation can be unexpectedly complicated. In order to appreciate this fact, consider the simple threegrain assemblage shown in figure 3 , which is considered to be part of a larger assemblage 
subjected to a remote tensile stress $\sigma_{\mathrm{e}}$ acting in the $y$-direction. The initial vertical load on all facets (facets $1-10, a, b)$ is $\sigma_{\mathrm{e}} A_{i}$, where $A_{i}$ is the projected area of facet $i$ on the $x-z$ plane. If the horizontal facet $a$ fails first, the vertical load $\sigma_{\mathrm{e}} A_{a}$ shed from this facet is equally distributed to the area contained in its adjacent oblique facets $1-3, b$, which belong to grain 1 , and facets $4-7$, which belong to grain 2 . Thus, the vertical load on these facets becomes

$$
P_{i}(1)=\sigma_{\mathrm{e}} A_{i}+\frac{\sigma_{\mathrm{e}} A_{a}}{8}
$$

where $i=1-3, b$ and 4-7. Here, the notation $P_{i}(k)$ denotes the vertical load on facet $i$ immediately after the occurrence of the $k$ th facet failure. Consequently, the index $k$ represents the time sequence of facet failures in the body. The vertical load on non-adjacent facets, such as facets 8,9 or 10 , remains unchanged. If facet $b$, which is common to grains 1 and 3 , subsequently fails, the load $P_{b}(1)$ will be equally redistributed over the area of the facets adjacent to $b$, i.e. facets $1, a, 3$ (grain 1) and facets 9, 8, 10 (grain 3). Note that, as a consequence of the second of the load-sharing rules, this load is not redistributed to the oblique facets 7 and 13 or to the vertical facets $11,12,14$, and 15, all of which are adjacent to facet 6 . From (8), the load to be redistributed will be

$$
\begin{aligned}
\Delta P_{i}^{b}=P_{b}(1)\left(\frac{1}{4 r_{A}+2}\right) & i=a, 8 \\
\Delta P_{i}^{b}=P_{b}(1)\left(\frac{r_{A}}{4 r_{A}+2}\right) & i=1,3,9,10
\end{aligned}
$$

where $r_{A}$ is the ratio of the component of the area of the oblique facet normal to the loading axis to that of the horizontal facet, i.e. $r_{A}=A_{\mathrm{o}} / A_{\mathrm{h}}$. For the present study, $r_{A}=1.5$. The notation $\Delta P_{i}^{b}$ denotes the initial adjustment to the load on facet $i$ due to the failure of facet $b$. However, facet $a$ has already failed, and cannot carry the load $\Delta P_{a}^{b}$. Consequently, it is necessary to further distribute $\Delta P_{a}^{b}$ to the facets which are adjacent to facet $a$, i.e. facets 1-7, $b$. The load to be distributed to these facets will be

$$
\Delta P_{i}^{a}=\frac{1}{8} \Delta P_{a}^{b}=\frac{1}{8}\left[P_{b}(1)\left(\frac{1}{4 r_{A}+2}\right)\right] \quad i=1-7, b .
$$

However, because facet $b$ has already failed, the load $\Delta P_{b}^{a} / 8$, in turn, must be redistributed to the facets adjacent to $b$, i.e. facets $1, a, 3,9,8,10$. Proceeding in this fashion leads directly to a recursive calculation for the vertical load on facets $1-10$, after both facet $a$ and $b$ have failed. For the present simple example, this takes the form of a geometric series. For example, for facets 1 and 3, with $r=\frac{1}{8}\left(1 /\left(4 r_{A}+2\right)\right)$ :

$$
\begin{aligned}
P_{i}(2)= & P_{i}(1)+\Delta P_{i}^{b}+r \Delta P_{i}^{b}+r^{2} \Delta P_{i}^{b}+r^{3} \Delta P_{i}^{b}+\Delta P_{i}^{a} \\
& +r \Delta P_{i}^{a}+r^{2} \Delta P_{i}^{a}+r^{3} \Delta P_{i}^{a}+\cdots \\
= & P_{i}(1)+\left(\Delta P_{i}^{b}+\Delta P_{i}^{a}\right) \sum_{n=0}^{\infty} r^{n} \\
= & P_{i}(1)+\left(\Delta P_{i}^{b}+\Delta P_{i}^{a}\right) /(1-r) .
\end{aligned}
$$

Summing the series for the remaining facets, the following expression is obtained

$$
P_{i}(2)=P_{i}(1)+\Delta P_{i}(2)
$$

where

$$
\Delta P_{i}(2)= \begin{cases}\left(\Delta P_{i}^{a}+\Delta P_{i}^{b}\right) /(1-r) & i=1,3 \\ \Delta P_{i}^{a} /(1-r) & i=2,4,5,6,7 \\ \Delta P_{i}^{b} /(1-r) & i=8,9,10 .\end{cases}
$$


It can be easily shown that the same load distribution will be obtained if the order of facet failure is reversed. Thus, the load-sharing scheme is dependent only on the geometrical position of the failed facets and not upon failure sequence.

Although it was possible to obtain closed-form expressions for the recursive expressions in this simple example, it can be quite difficult in more general situations. Hence, in numerical calculations, it was found necessary to redistribute the loads recursively until further redistributions resulted in a negligible change in facet stress.

The load-sharing rules proposed here undoubtedly result in an oversimplified estimate of the actual stress distribution. However, they at least roughly capture most of the relevant features of the load redistribution, and, in any case, a more accurate representation would be likely to increase the computational effort beyond the bounds of feasibility.

\subsection{Failure criterion}

A crucial part of the model is the failure criterion for the grain assemblage, for which there exist a number of possible choices. The one which will receive primary attention here, and which seems particularly reasonable within the context of the model, defines failure as the occurrence of a region consisting of a critical number of contiguous failed facets. Such a criterion assumes that, once a flaw of critical size forms, final failure ensues so rapidly that essentially all of the time up to final failure is taken up in forming the flaw. After some degree of numerical experimentation with an assemblage of 1729 grains and a representative set of parameters in the random facet failure model, the minimum number of contiguous failed facets to represent a flaw of critical size was taken to be 150 . To be more precise is quite difficult because of the rather complicated three-dimensional geometrical pattern of contiguous failed facets which can evolve. For flaw sizes containing at least this number of failed facets, it was found that the subsequent flaw size began to increase relatively rapidly, adding one or more failed facets on each time step. This value for the critical flaw size is, of course, to some extent arbitrary, and may depend to a degree upon the model parameters. However, it leads to reasonable agreement with experimental data, as will be discussed later.

It is worth noting that the use of this criterion could lead to failure as a result of a large flaw oblique to the loading axis. Although such failures are occasionally seen experimentally, the majority of failures seem to result from flaws normal to the loading axis. If one wishes to restrict failure to planes normal to the loading axis, an alternative failure criterion would be to define failure to be the occurrence of a critical number of failed facets within a 1- to 2-layer cross section of the grain assemblage normal to the loading axis. In this criterion, the number of failed facets, which occupy the same cross-sectional area within layers, are counted just once. Comparisons will be made between these two criteria.

Consideration was also given to adopting a failure criterion in which failure would be considered to have occurred when the grain assemblage had fractured into two separate pieces. Although this criterion has the advantage of being very straightforward, it was found to be computationally infeasible, owing to both the excessively long computation times required and the complicated three-dimensional topological considerations involved in determining when separation into two pieces had actually occurred. For example, a flaw running the width of the assemblage, which, in a two-dimensional array, would lead to failure under this criterion, would not do so in the present three-dimensional situation unless the entire thickness of the specimen was likewise failed. The determination of failure according to the 'two pieces' criterion is a difficult computational task. 


\section{Simulation}

Because of the complexity of the model described above, Monte Carlo simulation techniques were used to estimate its statistical behaviour. For each simulation, a random time-to-failure was assigned to each interior grain facet, using the CDF (5) for the standard exponential RVs and the transformation given in (6). Time was then advanced to the minimum facet time-to-failure, and the facet with this failure time was considered to have failed. Next, a search was conducted for all the facets adjacent to the failed facet, and the load supported by the failed facet redistributed to these facets, according to the rules described previously. The failure times for these facets were recalculated using (8). If the newly failed facet was adjacent to a previously failed facet, then the recursive load redistribution calculations outlined previously were carried out until the redistributed loads converged to within a specified tolerance. Finally, the assemblage was checked for overall failure, according to the prescribed failure criterion. If failure of the assemblage was not found to occur, then time was advanced to the next smallest facet failure time for the remaining intact facets, and the process was continued until the failure criterion was satisfied. To illustrate this rather complex procedure, an example is given in the appendix which captures most of the algorithm.

Because a sizeable amount of information is required to describe a large assemblage of grains, there are obvious memory constraints on the simulations. Not as obvious are execution time constraints, which proved to be the principal computational limitation, rather than memory. As facets fail, propagate and coalesce, there are more and more neighbouring facets to which the load can be redistributed. Typically, there was a fairly rapid initial period of individual facet failures until a sizeable contiguous flaw developed. This flaw then dominated the subsequent failure process, the increased normal stress on facets neighbouring the flaw being sufficiently large that, with few exceptions, they were the only facets to fail. As the size of the dominant flaw increased, there was a corresponding increase in the computational effort required to check for failure of adjacent facets, and to redistribute the stresses accordingly. Towards the end of each simulation, the computational time required to advance the failure process became quite large.

\section{Results}

For each set of the parameters $\alpha$ and $\rho$ chosen, 100 simulations were conducted as described in the previous section. The existence of a contiguous region composed of a critical number of failed facets was chosen as the failure criterion. For each set of parameters, the CDFs for the time-to-failure and the percentage of failed internal facets as a function of time were calculated. The fraction of failed facets were then later averaged over each of the simulations, as a function of time normalized by time-to-failure, to obtain an overall estimate.

Values of $\alpha=0.25,0.5$ and 1 were assumed, which correspond, respectively, to coefficients of statistical variation $(\mathrm{CVs})$ of $831 \%, 224 \%$ and $100 \%$ in the facet failure times. In addition, the ratio $\gamma / \sigma_{\mathrm{e}}$ was taken to be unity, where $\sigma_{\mathrm{e}}$ is the remote applied stress.

Although the parameter $\rho$ strictly controls the sensitivity of the grain boundary failure time to the facet normal stress, it must bear some approximate relation to the exponent in the power-law dependence normally observed between applied stress and failure time in time-dependent, intergranular failure in polycrystalline materials. Most metals, and some ceramics, have values of this exponent in the range 5-12. Moreover, there exists a fairly wide range of ceramics which have creep exponents in the range 1-3, e.g. [31-34]. 
Assuming that these materials obey the Monkman-Grant relation, as is the case with a good many, if not most, ceramic materials [35], a similar power-law dependence of stress upon failure time can be expected. Hence, values of $\rho=1,5,10$ and 15 are assumed.

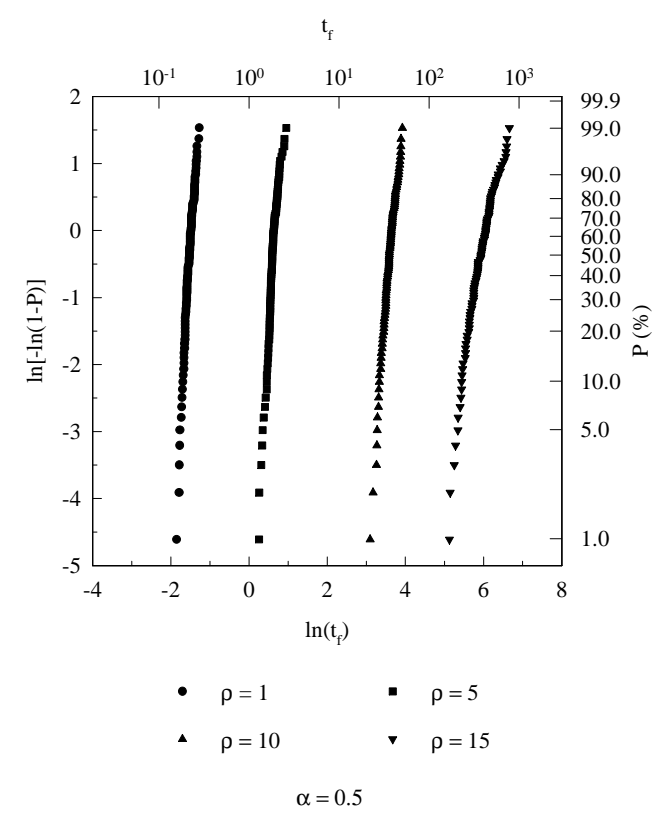

Figure 4. Simulated CDFs for normalized time-to-failure as $\rho$ varies, while $\alpha$ is fixed.

Figure 4 shows the computed CDFs for the probability of failure versus the normalized time-to-failure $t_{f} / \beta_{0}$, where $\beta_{0}=\left(\sigma_{\mathrm{e}} / \gamma\right)^{\rho}=1$, plotted on Weibull probability paper, for a matrix of 1729 grains. Figure 4 shows the parametric effect as $\rho$ varies while $\alpha$ is fixed at 0.5. Because the simulated data are nearly linear on Weibull paper, it is clear that the failure times are well approximated by a Weibull CDF. The median normalized times-to-failure are approximately $0.22,1.81,36.20$ and 379.89 for $\rho=1,5,10$ and 15 , respectively. Clearly, the power-law breakdown exponent $\rho$ has a significant effect on the time-to-failure. The CVs in the failure times are approximately $12 \%, 13 \%, 17 \%$ and $32 \%$ for $\rho=1,5,10$ and 15 , respectively, which is far less than the CV of $224 \%$ for the failure time of an isolated grain boundary for $\alpha=0.5$. As $\rho$ increases, the CV also increases, albeit somewhat slowly. It should be noted that when $\rho=15$, the Weibull CDF becomes a questionable approximation to the actual CDF. As $\rho$ increases, the scatter increases, and the Weibull approximation for the CDF for the time-to-failure is even less appropriate.

Figure 5 is similar to figure 4, except that here $\rho$ is held constant while the Weibull shape parameter $\alpha$ is allowed to vary. The median of the normalized times-to-failure are approximately $0.14,1.81$ and 9.69 for $\alpha=0.25,0.50$ and 1.00 , respectively. The CVs in the failure times are approximately $24 \%, 13 \%$ and $7 \%$ for $\alpha=0.25,0.50$ and 1.00 , respectively. Thus, as $\alpha$ decreases, so that the underlying scatter increases, the scatter in the times-to-failure also increases. Again, the CDFs are greatly influenced by the underlying shape parameter $\alpha$.

Figure 6 shows a plot of the fraction of failed internal grain boundary facets, as a function of time nondimensionalized by the time-to-failure, averaged over all of the appropriate simulations. It is clear that higher values of $\rho$ lead to the failure of a fairly 


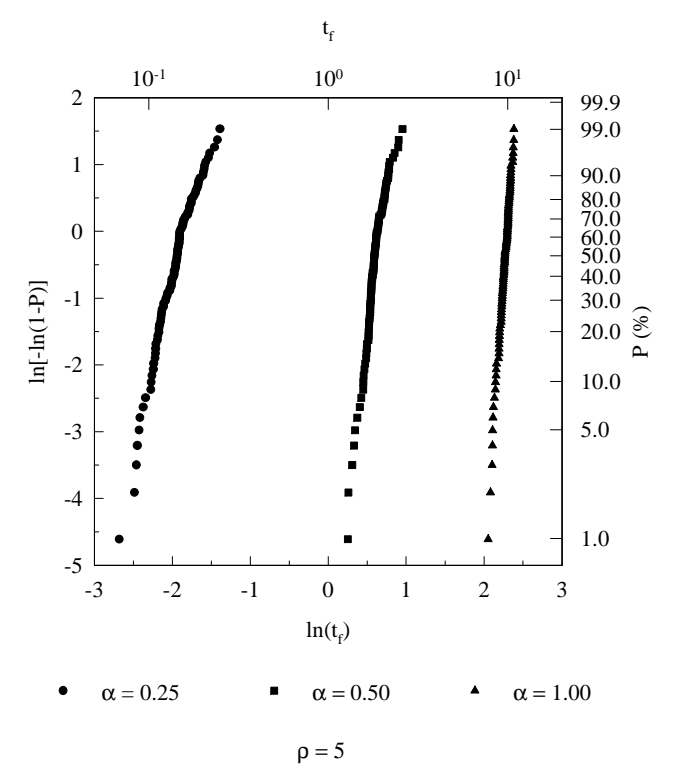

Figure 5. Simulated CDFs for normalized time-to-failure as $\alpha$ varies, while $\rho$ is fixed.

large number of facets early in the lifetime of the specimen, while the lower values of $\rho$ yield a relatively steady progression of facet failures throughout the lifetime of the specimen. This phenomenon results from the fact that higher values of $\rho$ lead to an increased sensitivity of facet failure times to grain boundary normal stress. Hence, facets normal to the applied stress, which have the higher initial stress, tend to fail prior to the oblique facets. This is evident in the nearly horizontal nature of the curve for $\rho=15$. After the normal stress on the oblique facets becomes sufficiently large due to the failure of surrounding normal facets, the oblique facets begin to make a substantial contribution to the failure process. In contrast, when $\rho$ is small, the damage progresses by the intermittent failure of both horizontal and oblique facets on a roughly equal basis. As an illustration of this phenomenon, the percentage of failed facets which are horizontal for one representative set of simulations is $31 \%, 60 \%, 74 \%$ and $90 \%$ for $\rho=1,5,10$ and 15 , respectively. The influence of $\alpha$ for fixed $\rho$ was relatively minor, with the averaged fraction of failed facets being primarily dominated by the power-law exponent $\rho$.

As was noted earlier, the critical flaw criterion is not the only possible failure criterion that one might consider. Another is that failure may be deemed to have occurred when a cross-sectional area normal to the loading axis becomes sufficiently weakened. Such a criterion effectively prevents failure due to an oblique flaw. Specifically, the matrix is assumed to have failed when the total number of failed facets within any 1- to 2-layer cross section exceeds 150 , with no restriction that the failed facets be contiguous. Note that, for the matrix of 1729 grains, the maximum number of facets in a layer is 581, excluding duplications. This criterion differs from the critical flaw criterion used previously, in that no critical flaw size is assumed. Moreover, with this criterion failure is restricted to planes normal to the loading axis, which is not the case with the critical flaw criterion. These two criteria approximately represent the extremes of the range of types of failure which have been observed experimentally, i.e. failure surfaces range from very irregular to essentially planar. Therefore, depending on the application, either of the criteria may be suitable. 


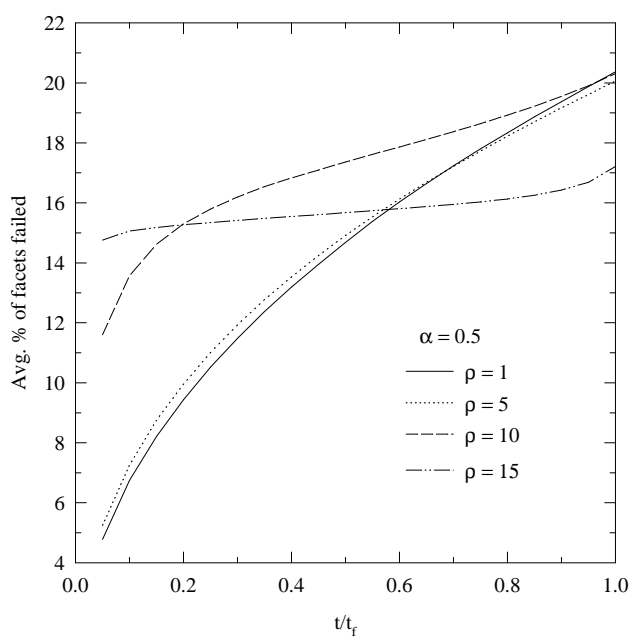

Figure 6. Ratio of failed grain boundary facets to total number of interior facets $v s$. fraction of total life.

Separate simulations using this failure criterion were conducted. The CDFs for the resulting times-to-failure using this criterion essentially have the same appearance as those shown in figures 4 and 5 . Nevertheless, the parameters of the CDFs are quite different. For $\rho=1,5,10$ and 15 , the median normalized times-to-failure are approximately $0.05,0.31$, 0.92 and 1.14, respectively, with the corresponding CVs being approximately $12 \%, 11 \%$, $13 \%$ and $13 \%$. Likewise, for $\alpha=0.25,0.50$ and 1.00 , the medians are approximately 0.01 , 0.31 and 0.97 , respectively, with corresponding $\mathrm{CVs}$ of $22 \%, 11 \%$ and $7 \%$. The times-tofailure are much smaller for this criterion than for the previous criterion. When 150 facets had failed in some 1- to 2-layer cross section, the maximum number of contiguous failed facets was typically less than 10 . The fraction of failed facets as a function of time is not very sensitive to the value of $\rho$ for this failure criterion. In fact, allowing for the lack of accuracy from the simulations, plots of $t / t_{f}$ against average fraction of failed facets for different values of $\rho$ are nearly identical. The influence of $\alpha$ is roughly the same for both criteria. The primary conclusion to be drawn from this comparison is that, not surprisingly, the failure behaviour is quite sensitive to the particular failure criterion employed. Thus, great care must be given to the selection of a realistic failure criterion.

The results discussed so far have been obtained using a perfectly-packed cubic array of $1729\left(10^{3}+9^{3}\right)$ grains. Naturally, the question of size effect arises. In order to investigate this question, separate sets of simulations have been conducted with arrays containing $189\left(5^{3}+4^{3}\right)$ grains, $559\left(7^{3}+6^{3}\right)$ grains and $6119\left(15^{3}+14^{3}\right)$ grains, for $\rho=5$ and $\alpha=0.25,0.50$ and 1.00. Figure 7 is a plot of matrix size versus the 63.21 percentile for the times-to-failure (the time by which $63.21 \%$ of the assemblages will have failed). At least for this range of sizes, the size effect may be estimated by a power-law relation of the form

$$
t_{f}=c N_{\mathrm{m}}^{-1 / \beta}
$$

where $N_{\mathrm{m}}$ is the total number of grains in the matrix, $c$ is an appropriate scaling constant and $\beta$ is the shape parameter which may be estimated from the slope of the lines on figure 7. It is interesting to note that the power-law size-effect scaling is typical of weakest-link behaviour in which the underlying CDF is a two-parameter Weibull CDF [36]. 


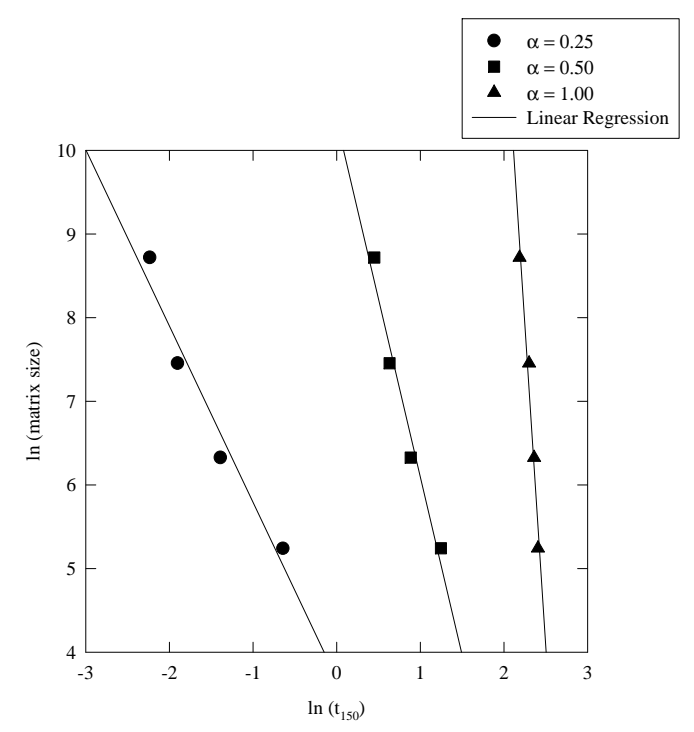

Figure 7. Size effect on 63.21 percentile of times-to-failure, failure defined by 150 contiguous failed facets.

Thus, it may be reasonable to approximate the central tendency of the time-to-failure by a Weibull CDF.

The weakest-link scaling for the central tendency of the time-to-failure suggests that the material may act in a weakest-link manner over the entire CDF; such behaviour has been observed for composite materials $[29,37]$. Let $W(t)$ be a nondegenerate CDF which is characteristic of the underlying probability structure of the material failure time. For composite materials, the equivalent $\mathrm{CDF}$ to $W(t)$ has been shown to be the limit distribution of a stochastically ordered sequence of CDFs. No such analysis exists for the current three-dimensional structure; however, the weakest-link assumption can be evaluated by considering the simulated CDFs from the four different sizes of arrays. Let $G_{N m}(t)$ be the $\mathrm{CDF}$ for the time-to-failure $T_{N_{\mathrm{m}}}$ of an array of $N_{\mathrm{m}}$ total grains. The weakest-link assumption is given by

$$
G_{N_{\mathrm{m}}}(t)=\operatorname{Pr}\left\{T_{N_{\mathrm{m}}} \leqslant t\right\}=1-[1-W(t)]^{N_{\mathrm{m}}}
$$

or by

$$
W(t)=1-\left[1-G_{N_{\mathrm{m}}}(t)\right]^{1 / N_{\mathrm{m}}}
$$

which is the reverse weakest-link scaling. Weibull probability paper is well suited to observing the behaviour of (10) or (11) because the standard Weibull transformation [37] implies that

$$
\ln \left[-\ln \left(1-G_{N_{\mathrm{m}}}(t)\right)\right]=\ln \left(N_{\mathrm{m}}\right)+\ln [-\ln (1-W(t))] .
$$

If $W(t)$ were known, then $G_{N_{\mathrm{m}}}(t)$ could be easily determined for any size $N_{\mathrm{m}}$. In fact, $W(t)$ and $G_{N_{\mathrm{m}}}(t)$ are simply related by shifting the vertical scale on the Weibull probability graph by $\ln \left(N_{\mathrm{m}}\right)$. Unfortunately, $W(t)$ is not known, and $G_{N_{\mathrm{m}}}(t)$ has been estimated only through simulations. Even so, rewriting (12),

$$
\ln [-\ln (1-W(t))]=-\ln \left(N_{\mathrm{m}}\right)+\ln \left[-\ln \left(1-G_{N_{\mathrm{m}}}(t)\right)\right]
$$


implies that $W(t)$, in turn, could be determined by suitably shifting the scale for $G_{N_{\mathrm{m}}}(t)$, if (10) or (11) is indeed applicable. Figure 8 shows the simulated CDFs for the four different sizes of arrays after the appropriate vertical scale shift. The similarity of the simulated values is striking. Each solid line is an estimation of $W(t)$ fit through the rescaled simulated values. The general trend is quite similar to that observed for composite materials [29]. However, this observation is purely empirical, and currently there is no way to describe $W(t)$ analytically. In reality, $W(t)$ is a complicated function of the three-dimensional geometry and load-sharing rule, the underlying probabilistic nature of the facets, and the assumed mechanism for facet failure. On the other hand, lacking any other means of extrapolation, the empirical estimations for $W(t)$ may be quite reasonable for large arrays.

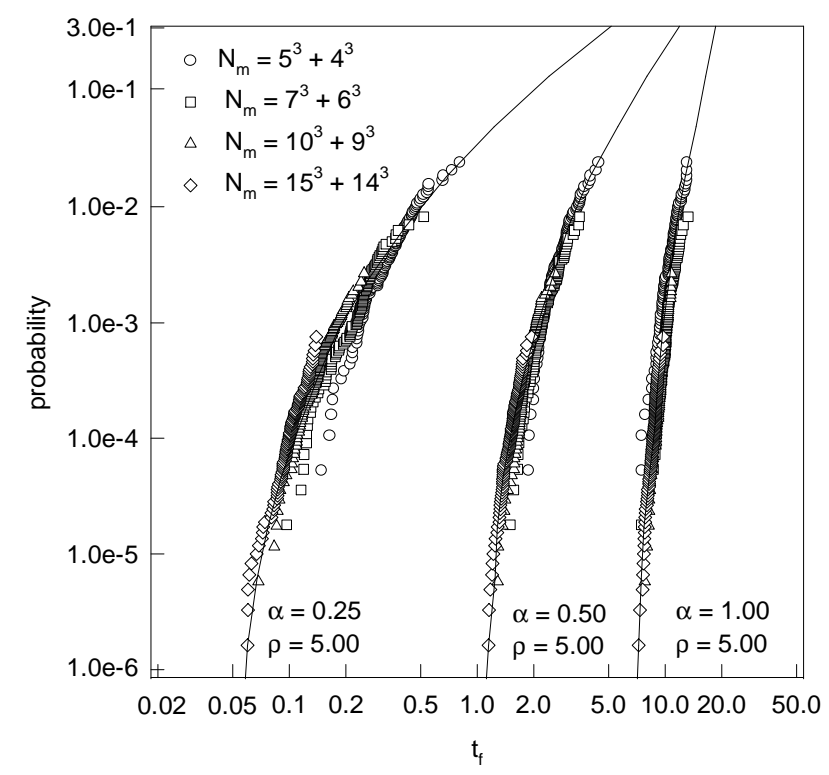

Figure 8. Weakest-link scaling of simulated CDFs and an estimate for the characteristic CDF $W(t)$.

\section{Discussion}

A probabilistic model has been developed for time-dependent intergranular failure in threedimensional polycrystalline arrays, a failure mode typically associated with materials in elevated-temperature service. The basic assumption of the model is that failure is governed by the grain boundary facet normal stress, and that the time-to-failure of each individual facet is a random function of this quantity. A simple approximate method for calculating the load shed by failed facets and a reasonable choice of failure criterion complete the model. Despite the simplicity of the ingredients, the result is a computationally complex model whose statistical behaviour can only be inferred through simulation techniques.

The most prominent of the predictions of the model is the scatter in the predicted timesto-failure. Calculated coefficients of variation for the failure times range from $7 \%$ up to $32 \%$. To compare these values with experimental observation, one may refer to the relatively small amount of replicated time-dependent failure data for metals and ceramics, e.g. [1,4]. A set of replicated creep rupture data for AISI type 316 stainless steel at $593^{\circ} \mathrm{C}$ [1], for 
example, exhibits coefficients of variations for the failure times of the order of $25-55 \%$. A more recent and extensive data set for hot isostatically pressed silicon nitride [4] indicates much higher coefficients of variation, which range from $67 \%$ to $205 \%$, depending upon the temperature and the stress level. Allowing for the fact that at least a fraction of the observed scatter must have resulted from random variations in experimental conditions, e.g. $[3,38]$, the predictions of the model appear to be in reasonable agreement with the type 316 stainless steel data, although indicating less scatter than that observed in the silicon nitride data. It is worth noting, however, that the inclusion of additional random features in the model, such as randomly-shaped grains, would be likely to increase the degree of scatter predicted by the model.

The average fraction of the failed facets as computed here is essentially identical to the $A_{c}$ damage parameter proposed by Cane and Townsend [39] and Shamas [40], apart from the fact that $A_{c}$ is typically measured on planar sections, while the quantity calculated here is derived from a full three-dimensional calculation. It is also closely related to the $\rho$ damage parameter of Riedel [41]. For values of $\rho$ in the range 1-5, the predicted variation of the average fraction of failed facets with time, as shown in figure 6 , bears a reasonable resemblance, both in a qualitative and a qualitative sense, to experimental data due to Shamas for the variation of $A_{c}$ versus time quoted by Riedel [41]. Of particular interest is the fact that the Shamas data indicate failure at a value of $A_{c}$ of approximately 0.4 , while the failure criterion adopted for the present model typically results in failure at a value of approximately 0.2 , regardless of the value of the parameter $\rho$ (except for very high $\rho$ values). Riedel [41] has estimated that an $A_{c}$ value of 0.2 corresponds to the expenditure of 75-90\% of the specimen lifetime. Thus, it is concluded that the critical flaw failure criterion adopted here results in predicted failure times that are somewhat below experimentally observed values, but not unreasonably so.

It is interesting to note that the model predicts the existence of a marked size effect upon times-to-failure. For the range of sizes considered, the failure times scale with size in a manner similar to that predicted by weakest-link failure models. However, the characteristic CDF, which depends on the three-dimensional geometry and failure mechanism, the material parameters, and the damage evolution, is entirely empirically estimated. Clearly more effort is warranted, if this observation is to be widely applicable.

Unfortunately, there does not appear to be an equivalent study using, for example, a regular hexagonal grain structure in two dimensions, with which the present work can be directly compared. Thus, it is difficult to make a direct assessment of the effects of threedimensionality in the present model. However, it is suspected that these effects are more likely to be quantitative than qualitative, and that, for example, the basic form of the failure time distribution would be altered little in a two-dimensional model. Nevertheless, fairly substantial differences in computed values may be expected. This supposition remains to be verified. On the other hand, this three-dimensional model introduces additional degrees of freedom in the growth of damage which cannot be captured in two-dimensional models. The load-sharing rule is necessarily more complex, and the failure criterion is not as straightforward as in a two-dimensional model. Again, these effects require further investigation.

Undoubtedly, the part of the proposed model most amenable to improvement is the approximate model adopted for the redistribution of the load shed from failed grain boundary facets to adjacent, unfailed facets. Here a simple load-sharing rule, similar to that used in the analysis of random fibre failures in composite materials, e.g. [28-30], has been used, and any possible effects of grain boundary sliding or grain deformability have been ignored. Thus, only the rough features of what must be in reality a very complicated 
process can be modelled. Unfortunately, more detailed descriptions of three-dimensional grain boundary facet stresses, such as those given by Anderson and Rice [12], and more recently by Rodin [42], still seem to be well beyond the bounds of analytic or computational feasibility for the complex, nonsymmetric situation considered here. In this regard, one may cite the recent results of van der Geissen and Tvergaard [11], who carried out detailed numerical studies of an irregular deforming grain structure with random intergranular cavitation. However, the system considered by these authors was limited to a $5 \times 6$ twodimensional structure. In contrast, the simplifications introduced in the present model allow one to consider systems consisting of thousands of three-dimensional grains. Moreover, the predictions of the model seem to be, in most cases, in reasonable accord with available experimental data. This leads one to believe that the simple model proposed here captures most of the gross trends exhibited by this complex phenomenon.

\section{Acknowledgment}

This work was supported by the National Science Foundation under grant no DMR-9102093.

\section{Appendix}

Attention is focused upon the string of horizontal and oblique facets shown in figure A1, in the sequence h-o-h-o-h and numbered from left to right. Until the first facet fails, each facet supports a constant load $P_{i}(0)$ given by $P_{i}(0)=\sigma_{\mathrm{e}} A_{i}$, where $\sigma_{\mathrm{e}}$ is the remote stress and $A_{i}$ is the horizontal projection of the area of facet $i$. With the assumed loading orientation that is shown in figure 2 , we have that $\sigma_{\mathrm{h}}=\sigma_{\mathrm{e}}$ and $\sigma_{\mathrm{o}}=\sigma_{\mathrm{e}} / 3$. The simulation algorithm proceeds as follows:

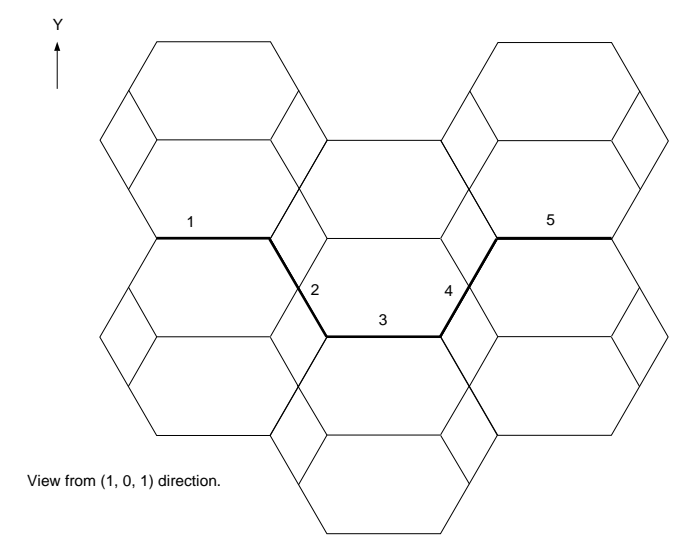

Figure A1. Five contiguous facets to demonstrate simulation procedure.

(1) Simulate the standard random exponential variables $Z_{w}$ associated with these facets. Let $u_{i}$ be a random number selected from the uniform CDF on the unit interval. Then $Z_{i}=-\ln \left(1-u_{i}\right)$. Assume that this process yields the five values $Z_{1}=1.37, Z_{2}=$ $0.97, Z_{3}=1.93, Z_{4}=1.03$ and $Z_{5}=3.58$.

(2) The key to the simulation is the solution for the appropriate time-to-failure in terms of the simulated standard exponential RVs and the hazard function as given in (6). The 
primary equation is

$$
\left(Z_{i}\right)^{(1 / \alpha)}=\int_{0}^{T_{w}\left(i \mid i_{1}, \ldots, i_{j}\right)}\left(\frac{\sigma_{w}(s)}{\gamma}\right)^{\rho} \mathrm{d} s
$$

where $T_{w}\left(i \mid i_{1}, i_{2}, \ldots, i_{j}\right), w=\mathrm{h}$ (horizontal) or o (oblique), is the time-to-failure of facet $i$, given that facets $i_{1}, i_{2}, \ldots, i_{j}$, which lead to an increase in load on facet $i$, have failed. Prior to the first facet failure, the above equation can be solved to obtain

$$
T_{w}(i)=\left(Z_{i}\right)^{(1 / \alpha)}\left(\frac{\gamma}{\sigma_{w}}\right)^{\rho}= \begin{cases}\left(Z_{i}\right)^{(1 / \alpha)}\left(\frac{\gamma}{\sigma_{\mathrm{e}}}\right)^{\rho} & w=\mathrm{h} \\ \left(Z_{i}\right)^{(1 / \alpha)}\left(\frac{\gamma}{\sigma_{\mathrm{e}} / 3}\right)^{\rho} & w=\mathrm{o} .\end{cases}
$$

The first time-to-failure $T_{1}$ is the minimum of all of the computed times above, i.e.

$$
T_{1}=\min _{1 \leqslant i \leqslant N}\left(T_{w}(i)\right)
$$

where $N$ is the total number of facets. In order to complete the numerical example, assume that $\alpha=1 / 3$ and $\rho=2.0$. Recall that the time can be nondimensionalized by setting $\left(\sigma_{\mathrm{e}} / \gamma\right)^{\rho}=1$, and this is used throughout this example. Thus, for the sequence of five horizontal and oblique facets, $T_{\mathrm{h}}(1)=2.57, T_{\mathrm{o}}(2)=8.21, T_{\mathrm{h}}(3)=7.19, T_{\mathrm{o}}(4)=9.83$, and $T_{\mathrm{h}}(5)=45.88$. Hence, $T_{1}=2.57$, and it occurs at facet 1 , which is horizontal.

(3) Facet 1 is now assumed to have failed at time $T_{1}$, and the load carried by this facet is redistributed to the eight adjacent oblique facets. Recall that $A_{\mathrm{o}} / A_{\mathrm{h}}=1.5$. Thus, the vertical load on facet 2 becomes

$$
P_{2}(1)=P_{2}(0)+P_{1}(0)\left(\frac{A_{\mathrm{o}}}{8 A_{\mathrm{o}}}\right)=\sigma_{\mathrm{e}}\left(A_{\mathrm{o}}+\frac{A_{\mathrm{h}}}{8}\right)=\frac{13}{12} \sigma_{\mathrm{e}} A_{\mathrm{o}}
$$

and the normal stress on facet 2 becomes

$$
\sigma_{2}(1)=\frac{1}{3}\left(\frac{P_{2}(1)}{A_{\mathrm{o}}}\right)=\frac{13}{12} \sigma_{\mathrm{o}}=\frac{13}{36} \sigma_{\mathrm{e}} .
$$

Note that the normal stress on facets 3,4 and 5 remains the same, since they are not adjacent to any failed facet.

(4) The next step is to recompute the time-to-failure for those facets which experience an increase in load. This computation must take into account both the magnitude of the load increase and the times at which these increases occurred. For those facets which do not experience an increase in load, the times-to-failure remain the same as previously computed. However, the time-to-failure for the facets that experience an increase in load must be reduced appropriately. Thus, the solution from step (2) becomes, from equation (8),

$$
T_{w}\left(i \mid i_{1}\right)=T_{1}+\left(Z_{i}^{1 / \alpha}-T_{1}\left(\frac{\sigma_{i}(0)}{\gamma}\right)^{\rho}\right)\left(\frac{\gamma}{\sigma_{i}(1)}\right)^{\rho} .
$$

For the present example, the new time-to-failure $T_{0}(2 \mid 1)$ may be found by computing

$$
T_{\mathrm{o}}(2 \mid 1)=2.57+\left(0.97^{3}-2.57(1 / 3)^{2}\right)(36 / 13)^{2}=7.38 .
$$

The second time-to-failure $T_{2}$ is the minimum of all of the facet failure times, including both those which have experienced increases in load and those that have not. Hence $T_{2}=7.19$ from facet 3 in the example.

(5) The new stress distribution is computed again as in step (3) after facet 3 has failed at $T_{2}$, and the process is repeated. The vertical loads on the three remaining facets are

$$
P_{2}(2)=P_{2}(1)+P_{3}(1)\left(\frac{A_{\mathrm{o}}}{8 A_{\mathrm{o}}}\right)=\sigma_{\mathrm{e}}\left(A_{\mathrm{o}}+\frac{A_{\mathrm{h}}}{4}\right)=\frac{7}{6} \sigma_{\mathrm{e}} A_{\mathrm{o}}
$$




$$
\begin{aligned}
& P_{4}(2)=P_{4}(1)+P_{3}(1)\left(\frac{A_{\mathrm{o}}}{8 A_{\mathrm{o}}}\right)=\sigma_{\mathrm{e}}\left(A_{\mathrm{o}}+\frac{A_{\mathrm{h}}}{8}\right)=\frac{13}{12} \sigma_{\mathrm{e}} A_{\mathrm{o}} \\
& P_{5}(2)=P_{5}(1)=\sigma_{\mathrm{e}} A_{\mathrm{h}}
\end{aligned}
$$

and the corresponding normal stresses are

$$
\sigma_{2}(2)=\frac{7}{6} \sigma_{\mathrm{o}}=\frac{7}{18} \sigma_{\mathrm{e}} \quad \sigma_{4}(2)=\frac{13}{12} \sigma_{\mathrm{o}}=\frac{13}{36} \sigma_{\mathrm{e}} \quad \sigma_{5}(2)=\sigma_{\mathrm{h}}=\sigma_{\mathrm{e}} .
$$

The adjusted time-to-failure for facet 2 is found from (8) as

$$
\begin{aligned}
T_{\mathrm{o}}(2 \mid 1,3) & =T_{2}+\left[Z_{2}^{1 / \alpha}-T_{1}\left(\frac{\sigma_{2}(0)}{\gamma}\right)^{\rho}-\left(T_{2}-T_{1}\right)\left(\frac{\sigma_{2}(1)}{\gamma}\right)^{\rho}\right]\left(\frac{\gamma}{\sigma_{2}(2)}\right)^{\rho} \\
& =7.19+\left[0.97^{3}-2.57(1 / 3)^{2}-(7.19-2.57)(36 / 13)^{2}\right](18 / 7)^{2} \\
& =7.35
\end{aligned}
$$

Likewise,

$$
T_{0}(4 \mid 3)=7.19+\left(1.03^{3}-7.19(1 / 3)^{2}\right)(36 / 13)^{2}=9.44
$$

The third time-to-failure $T_{3}$ is the minimum of $\{7.35,9.44,45.88\}$, which corresponds to facet 2 . Again, there is load redistribution after facet 2 has failed at $T_{3}$, and the failure times are recomputed for those facets with the increased loads. The increase in vertical load on facet 4 is given by

$$
P_{4}(3)=P_{4}(2)+\Delta P_{4}(3)
$$

and a recursive process of load redistribution, similar to that described previously, yields

$$
\Delta P_{4}(3)=\Delta P_{4}^{3} /(1-2 r)
$$

where $\Delta P_{4}^{3}=r P_{2}(2)$ and now $r=\frac{1}{8}\left(A_{\mathrm{h}} /\left(4 A_{\mathrm{o}}+2 A_{\mathrm{h}}\right)\right)=\frac{1}{64}$. Thus, the normal stress on facet 4 becomes

$$
\sigma_{4}(3)=\frac{13}{12} \sigma_{\mathrm{o}}+\frac{\sigma_{\mathrm{o}}\left(\frac{7}{6}\right)\left(\frac{1}{64}\right)}{1-2\left(\frac{1}{64}\right)}=\frac{205}{186} \sigma_{\mathrm{o}}=\frac{205}{558} \sigma_{\mathrm{e}}
$$

while the stress on facet 5 remains the same. The adjusted time-to-failure for facet 4 is given by

$$
\begin{aligned}
T_{\mathrm{o}}(4 \mid 3,2) & =T_{3}+\left[Z_{4}^{1 / \alpha}-T_{2}\left(\frac{\sigma_{4}(0)}{\gamma}\right)^{\rho}-\left(T_{3}-T_{2}\right)\left(\frac{\sigma_{4}(2)}{\gamma}\right)^{\rho}\right]\left(\frac{\gamma}{\sigma_{4}(3)}\right)^{\rho} \\
& =7.35+\left[1.03^{3}-7.19(1 / 3)^{2}-(7.35-7.19)(13 / 36)^{2}\right](558 / 205)^{2} \\
& =9.37 .
\end{aligned}
$$

Observe that $\sigma_{4}(1)$ does not appear in the above equation. This is because facet 1 , which was the first to fail, does not cause any further increase in the load on facet 4.

\section{References}

[1] Garafalo F, Whitmore R W, Domis W F and Von Gemmingen F 1961 Trans. Metall. Soc. AIME 221310

[2] Walles K F A 1967 Random and Systematic Factors in the Scatter of Creep Data C.P. No. 935 (London: Aeronautical Research Council, HMSO)

[3] Farris J P, Lee J D, Harlow D G and Delph T J 1990 Metall. Trans. 21A 345

[4] Menon N M, Fang H T, Wu D C, Jenkins M G, Ferber M K, More K L, Jubbard C R and Nolan T A 1994 J. Am. Cer. Soc. 771217

[5] Liu T S, Fields R J, Fariborz S J, Harlow D G and Delph T J 1988 Acta Metall. 362481

[6] Fariborz S J, Harlow D G and Delph T J 1985 Acta Metall. 331

[7] Chan K S and Page R A 1987 Metall. Trans.18A 1843 
[8] Yu J and Chung J O 1990 Acta Metall. 381432

[9] Xiao D, Harlow D G and Delph T J 1992 Phil. Mag. A 6571

[10] Riesch-Oppermann H and Brückner-Foit A 1994 Probabilistic Structural Mechanics: Advances in Structural Reliability Methods ed P D Spanos and Y-T Wu (Berlin: Springer) pp 442-54

[11] van der Giessen E and Tvergaard V 1994 Modelling Simul. Mater. Sci. Eng. 2721

[12] Anderson P E and Rice J R 1985 Acta Metall. 33409

[13] Ghahremani F 1980 Int. J. Solids Struct. 16847

[14] Cocks A C F and Ashby M F 1982 Prog. Mater. Sci. 27189

[15] Gilbert E N 1962 Ana. Math. Stat. 33958

[16] Green P J and Sibson R 1978 The Computer J. 21168

[17] Le Caër G and Ho J S 1990 J. Phys. A: Math. Gen. 233279

[18] Horálek V 1990 Mater. Char. 25263

[19] Tipper J C 1990 Information Processing Letters 34155

[20] Ito O and Fuller E R Jr 1993 Acta Metall. Mater. 41191

[21] Dib M W and Rodin G J 1993 J. Mech. Phys. Solids 41725

[22] Riedel H 1977 Fracture at High Temperatures (Berlin: Springer)

[23] Nix W D, Earthman J C, Eggeler G and Ilschner B 1989 Acta Metall. 371067

[24] Larson H J and Shubert B O 1979 Probabilistic Models in Engineering Sciences, Volume II: Random Noise, Signals and Dynamic Systems (New York: Wiley)

[25] Coleman B D 1958 J. Appl. Phys. 29968

[26] Phoenix S L 1978 Int. J. Fract. 14327

[27] Phoenix S L and Tierney L J 1983 Engr. Fract. Mech. 18193

[28] Harlow D G and Phoenix S L 1981 Int. J. Fract. 17347

[29] Harlow D G and Phoenix S L 1981 Int. J. Fract. 17601

[30] Harlow D G and Yukich J E 1993 Stoc. Proc. \& Appl. 44141

[31] Cannon R M and Coble R L 1975 Deformation of Ceramic Materials (New York: Plenum) p 61

[32] Dimos D and Kolstedt D L 1987 J. Am. Cer. Soc. 70531

[33] Parhasarathy T A, Mah T I and Keller K 1992 J. Am. Cer. Soc. 751756

[34] Robertson A G, Wilkinson D S and C Ceres C A 1991 J. Am. Cer. Soc. 74915

[35] Wiederhorn S H, Fields B A and Hockey B J 1993 Proc. Int. Conf. Fundamentals Frac. (Fukushima, Japan)

[36] Jayatilaka A de S and Trustrum K 1977 J. Mat. Sci. 121426

[37] Harlow D G and Phoenix S L 1978 J. Composite Mater. 12314

[38] Hayhurst D R 1974 Int. J. Mech. Sci. 16829

[39] Cane B J and Townsend R D 1985 Fracture and Flow at Elevated Temperatures ed R Raj (Metals Park, OH: ASM) p 149

[40] Shamas M S 1988 Inter. Conf. on Life Extension and Refurbishment of Steam Plants (Essen, Arnheim, Palo Alto: VGB, KEMA, EPRI) p 238

[41] Riedel H 1989 Int. J. Pres. Ves. \& Piping 39119

[42] Rodin G J 1995 J. Appl. Mech. 621 